\title{
Towards quantitative scales of lithophilicity, chalcophilicity and hydrophilicity using statistical correlations among the mineral- forming elements
}

\author{
Daniel R Hummer, ${ }^{1}$ Xiaogang Ma,${ }^{2}$ XIAng Que,${ }^{3}$ \\ SHUANG ZHANG ${ }^{4}$ CHAO LiU, ${ }^{4}$ ROBERT H. HAZEN, ${ }^{4}$ \\ JOSHUA J GOLDEN, ${ }^{5}$ ROBERT T DOWNS ${ }^{5}$ \\ ${ }^{1}$ School of Earth Systems and Sustainability, Southern Illinois \\ Univ., Carbondale, IL 62901 \\ ${ }^{2}$ Dept. of Comp. Science, Univ. of Idaho, Moscow, ID 83844 \\ ${ }^{3}$ Dept. of Comp. Engineering, Fujian Agriculture and Forestry \\ Univ., Fuzhou, China \\ ${ }^{4}$ Geophysical Laboratory, Carnegie Institution for Science, \\ Washington, DC 20015 \\ ${ }^{5}$ Dept. of Geosciences, Univ. of Arizona, Tucson, AZ 85721
}

The mineralogy of terrestrial planets is governed not only by size, bulk composition, and geochemical processes, but also by the way its constituent elements parse themselves into mineral species. To quantify mineral-forming behavior in Earth's naturally occurring elements, we used a mineral database (rruff.info/ima as of 5/15/2019) to conduct a statistical analysis of the number of mineral species containing both elements $X$ and $\mathrm{Y}$ for every possible $\mathrm{X}-\mathrm{Y}$ pair (and $\mathrm{X}, \mathrm{Y}$ and $\mathrm{Z}$ for every possible $X-Y-Z$ triplet) among all 72 mineral-forming elements occurring in 5477 known mineral species. The frequency with which an element pair or triplet occurred together in the nominal formula of a mineral was compared with the expected frequency if elements were distributed among mineral species randomly. The resulting correlations and anti-correlations ranged from $\sim 1$ to $10^{-308}$ (as measured by their $p$-value in a chi-squared test for element pairs, and a Cochran-Mantel-Haenszel test [1] for element triplets). Every element $\mathrm{X}$ yielding statistically significant results showed either strong correlation or strong anti-correlation for the triplet $\mathrm{X}-\mathrm{O}-\mathrm{H}$, indicating that elements conform to a binary hydrophilic-hydrophobic classification according to their preference for forming hydrous vs. anhydrous minerals, respectively. Moderate to strong correlations of most elements with either $\mathrm{O}$ or $\mathrm{S}$ conform exceptionally well to Goldschmidt's qualitative classification of elements as lithophile or chalcophile. [2] The strengths of these correlations and anti-correlations, as measured by their chi-squared $p$ values, correlates well with other measures of geochemical behavior and forms a basis for the first quantitative scales of lithophilicity and chalcophilicity.

[1] Mantel, M. (1963) J. Am. Stat. Assoc. 58, 690-700.

[2] Goldschmidt, V. (1937) J. Chem. Soc. 655-673. 